\title{
Klebsiella pneumonia-induced prostate abscess: How to work it up?
}

\author{
Jae Hoen Kim, MD, PhD;* Won Jae Yang, MD, PhD;* Tae Hyong Kim, MD, PhD \\ "Department of Urology, Soonchunhyang University, College of Medicine, Seoul, South Korea; Infection Urology, Soonchunhyang University, College of Medicine, Seoul, South Korea
}

Cite as: Can Urol Assoc J 2014;8(11-12):e841-4. http://dx.doi.org/10.5489/cuaj.2155 Published online November 24, 2014.

\section{Abstract}

Introduction: Klebsiella pneumonia (KP) is related to a metastatic phenomenon from the originally affected primary organ. About $28 \%$ of patients with pyogenic liver abscess arising from KP suffer from metastatic complications. This study was done to define the clinical features of KP-induced prostate abscess.

Methods: A total of 14 patients were diagnosed with prostate abscess based on clinical, laboratory examination and abdominopelvic computed tomography (CT) scan from 2007 to 2013.

Results: Among these 14 patients, KP was the dominant causative microorganism in 6 patients $(42.9 \%)$, followed by Esherchia coli in 2, Pseudomonas aeroginosa in 1, methicillin-resistant Staphyolcoccus aureus in 1, and no growth in either the urine or blood culture in 4 . Four $(66.7 \%)$ of the $6 \mathrm{KP}$ induced-prostate abscess had other concurrent abscess sites besides the prostate: liver in 3, kidney in 1, and perianal area with endogenous endophthalmitis that ended in loss of vision in 1 patient.

Conclusions: We report on the clinical features of KP-induced prostate abscess based on a small number of patients, which is the main limitation of our study. We believe that if the causative organism of a prostate abscess was $\mathrm{KP}$, more workup would be needed to rule out the presence of an abscess in other organs, especially in the liver. Abdominopelvic CT scan would be a proper imaging modality.

\section{Introduction}

Prostate abscess is rare mainly due to the development of broad-spectrum antibiotics which have dramatically reduced its incidence. ${ }^{1}$ Most prostate abscesses present during the fifth and sixth decades of life and they are usually secondary to bacterial prostatitis. ${ }^{2}$ It is primarily identified in patients with pre-existing medical conditions, indwelling catheters, infravesical obstruction, instrumentation of the lower urinary tract, diabetes, liver disease, and chronic renal failure on dialysis. It is also present in immunosuppressed patients, patients with internal prostheses (such as heart valves, joints, or penile implant), patients with acute and chronic bacterial prostatitis, and patients with a recent prostate biopsy. ${ }^{3,4}$

Prostate abscess formation, which was primarily caused by Neisseria gonorrhea (75\% of cases) in the pre-antibiotic era, is now predominantly caused by Esherchia coli (in $60 \%$ to $80 \%$ of cases). ${ }^{5}$ Other significant pathogens include Pseudomonas species, Staphylococcus species, and occasionally obligate anaerobic bacteria. ${ }^{6}$ Very few references exist about this pathology. ${ }^{1}$ The prevalence of Klebsiella pneumonia (KP)-induced prostate abscess varies with geographic area. In Spain, there was no KP observed in the 29 cases of prostate abscess, ${ }^{1}$ whereas KP was the number one causative organism of prostate abscess $(10 / 17,58.8 \%$ ) in a KP-endemic area, such as South Taiwan. ${ }^{7} \mathrm{KP}$ is the second dominant organism (17.3\%), following Eshcerchia coli $(40.4 \%)$, in prostate abscess in South Korea. ${ }^{8}$

KP belongs to the family Enterobacteriaceae, which are rod-shaped, gram-negative bacteria with a prominent polysaccharide capsule. This capsule encases the entire cell surface, accounts for the large appearance of the organism on Gram stain, and provides resistance against many host defense mechanisms. ${ }^{9} \mathrm{KP}$-induced liver abscess has been reported with increasing frequency in East Asian countries, especially in Taiwan and South Korea, in the past 3 decades. ${ }^{10,11}$ Patients with pyogenic liver abscess often have high rates of bacteraemia and subsequent hematogenous spread complications; and these sequelae are more frequent when KP is the causative agent. ${ }^{12}$ About $28 \%$ of patients with pyogenic liver abscess arising from KP suffered from hematogenous spread complications; hematogenous spreading prostate abscess was reported in 11/9839 KP-induced pyogenic liver abscesses. ${ }^{13}$ Also, KP has been the leading causative microorganism of emphysematous prostatic abscess, which is the lethal form of prostatic abscess. ${ }^{14}$ This study was performed to reveal the clinical characteristics of KP-induced prostate abscess. 
Kim et al.

\section{Methods}

After obtaining institutional review board approval, we examined all cases of prostate abscess at our institution from 2007 to 2013 via a retrospective chart review of inpatient records. From 2007, an electronic data system was adapted and all patients with acute prostatitis or prostate abscess were included and reviewed. Patients who had KP as the causative organism were selected. Prostate abscess was diagnosed based on clinical, laboratory examination and abdominopelvic computed tomography (CT) scan.

\section{Results}

A total of 14 patients were diagnosed with prostate abscess. The mean age was 72 years. Among the 14 patients, KP was the dominant microorganism in 6 patients $(42.9 \%), 2$ had Esherchia coli (14.3\%), 1 had Pseudomonas aeroginosa (7.1\%), 1 had methicillin-resistant Staphyolcoccus aureus $(7.1 \%)$, and 4 had no growth in either urine or blood culture (28.6\%).

Among the 6 patients with KP-induced prostate abscess, $4(66.7 \%)$ had other abscess focus besides the prostate: 3 in the liver (Cases 1,2 and 3), 1 in the kidney (Case 3), and 1 in the perianal area who developed loss of vision due to hematogenous spreading KP-induced endogenous endophthalmitis (Case 4) (Table 1). Case 1 had diabetes mellitus and liver cirrhosis for several years. He visited our clinic for acute urinary retention, pain between the scrotum and anus, and spiking fever. Any specific sign related with liver abscess was not found. Under the impression of acute prostatitis, relevant laboratory examinations were done. An abdominopelvic CT scan revealed concurrent prostate and liver abscess (Fig. 1). Likewise, Case 2 showed concurrent prostate and liver abscess in the abdominopelvic CT scan (Fig. 2). Case 2 also demonstrated signs and symptoms related to lower urinary tract infection. The liver abscess was cured with percutaneous drainage and proper antibiotics. Case 3 had multiple microabscesses in the liver and both kidneys (Fig. 3). Case 4 had a prostate and perianal abscess resulting in bacteremia. He complained of ophthalmologic symptoms and was diagnosed with endogenous endophthalmitis, which eventually ended in blindness despite critical care including vitrectomy. Except for Case 4, the other 5 patients were cured without any complication.

Among the 8 patients with prostate abscess wherein KP was not isolated, 1 (12.5\%) had liver abscess, but the urine or blood culture failed to yield a causative organism; this may be due to the fact that the patient was given antibiotics at a local hospital prior to the laboratory workup.

\section{Discussion}

The clinical presentation of a prostate abscess is usually non-specific and can vary from fever, rectal tenesmus or acute urinary retention; this variation makes it difficult to accurately confirm the diagnosis. ${ }^{2}$ Routine laboratory studies and prostate-specific antigen may demonstrate findings consistent with urinary tract infection or prostate cancer. Urine cultures may be negative, and may become positive when the abscess opens into the urethra or bladder. ${ }^{15}$

Initially, some prostate microabscesses can appear and if they do not respond adequately to antibiotic treatment or if no treatment is started; this situation can lead to the onset of prostate abscesses of different dimensions. ${ }^{1}$ Such abscesses usually are complications of acute bacterial prostatitis that have either not been treated or been inappropriately treated. ${ }^{16}$ Although symptoms overlap with those of acute prostatitis, an abscess must be suspected when symptoms do not resolve after 48 hours of intravenous antibiotic therapy. Despite the difficulty involved, it is very important to distinguish between acute prostatitis and prostate abscess, since the therapeutic approach for each is different. ${ }^{16}$ Since the diagnosis is often difficult to confirm by history and physical

Table 1. Characteristics of Klebsiella pneumonia-induced prostate abscess

\begin{tabular}{|c|c|c|c|c|c|c|}
\hline & Case 1 & Case 2 & Case 3 & Case 4 & Case 5 & Case 6 \\
\hline Age & 76 & 67 & 78 & 66 & 64 & 66 \\
\hline Chief complaint & AUR, Fever & AUR, Fever & AUR, Fever & Visual disturbance & Dysuria, Fever & Dysuria, Fever \\
\hline Comorbid disease & DM, HiBP, CVA & HiBP & None & None & DM, HiBP & HiBP \\
\hline $\mathrm{U} / \mathrm{C}$ & $\mathrm{KP}$ & $\mathrm{KP}$ & $\mathrm{KP}$ & $\mathrm{KP}$ & $\mathrm{KP}$ & None \\
\hline$B / C$ & None & None & $\mathrm{KP}$ & None & $\mathrm{KP}$ & $\mathrm{KP}$ \\
\hline PSA (ng/mL) & 74.97 & 33.38 & 72.60 & 12.73 & 13.01 & 11.04 \\
\hline Involved sites & Liver & Liver, Lung & Liver, Kidney, Lung & Eye, Perianus & None & None \\
\hline Procedures & $\begin{array}{l}\text { Cystostomy } \\
\text { TURP }\end{array}$ & $\begin{array}{c}\text { TURP } \\
\text { PCD (liver) }\end{array}$ & $\begin{array}{c}\text { TURP } \\
\text { Ceftriaxone }\end{array}$ & $\begin{array}{l}\text { I \& D (perianus) } \\
\text { Vitrectomy (eye) }\end{array}$ & None & None \\
\hline Antibiotics & Ciprofloxacin & Levofloxacin & Ceftriaxone & Ciprofloxacin & Ciprofloxacin & Ciprofloxacin \\
\hline Course & Recover & Recover & Recover & Sight loss & Recover & Recover \\
\hline
\end{tabular}

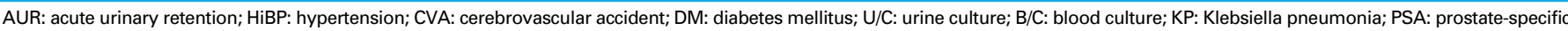
antigen; TURP: transurethral resection of prostate; PCD: percutaneus drainage; I \& D: incision and drainage. 


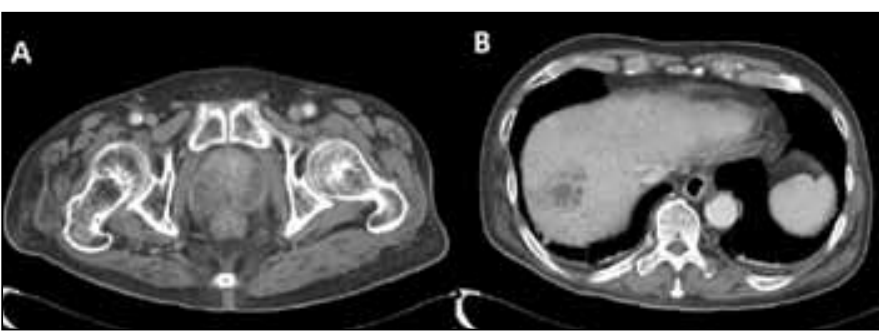

Fig. 1. A: Diffuse enlargement of prostate and ill-defined low density of prostate peripheral gland. B: About $3.7-\mathrm{cm}$ size peripheral thick enhancing cystic lesion in right lobe dome of liver.

examination alone, prostatic images (transrectal ultrasound, CT or magnetic resonance imaging) can be important in the diagnosis and management. ${ }^{17}$ In these cases, the best imaging modality is a CT of the abdomen and pelvic area, which can detect extraprostatic penetration, the extent of the pelvic inflammatory process and other possible infectious sources. ${ }^{18}$

Prompt medical and surgical management of prostatic abscess is crucial to prevent progression to sepsis and death. ${ }^{19}$ The management options include transrectal ultrasonography-guided aspiration, transurethral incision over the abscess, transurethral deroofing of the abscess cavity, or formal transurethral resection of prostate. ${ }^{20}$ No management algorithms are currently available to guide physicians in the surgical drainage, and the decision is usually based on physician preference.

Half of our patients affected by KP had concurrent liver abscess. Before the 1980s, the organism most commonly isolated from liver abscesses was Escherichia coli, but in Taiwan and Korea there has been a shift to KP, which is now highly endemic and the major cause of community-acquired pyogenic infections. ${ }^{9-13}$ Pope and colleagues reported that a similar etiological shift, like one seen in our cases, is now occurring in the United States. ${ }^{21}$ About $28 \%$ of patients with pyogenic liver abscess due to KP also suffer from metastatic complications, and the prostate can be involved. ${ }^{13}$ It is logical to postulate that the prostate was not the primary site, but secondary to the pyogenic liver abscess with KP in our cases. Authors hypothesized that primary pyogenic liver abscess with $\mathrm{KP}$, although rare, is more common that primary prostate abscess with KP. Our patients, however, visited the urology clinic due to their lower urinary tract symptoms and fever, and the liver abscess was detected after performing the abdominopelvic CT scan.

Endogenous endophthalmitis is a sight-threatening ophthalmic emergency that requires vitrectomy unless prompt treatment is undertaken. It is usually caused by hematogenous spread of an infection from a distant endogenous focus, mostly from the liver. We treated a patient with endogenous endophthalmitis who also had KP-induced prostate and perianal abscesses. Clinicians should entertain the possibility of endogenous endophthalmitis if a patient with a urologic

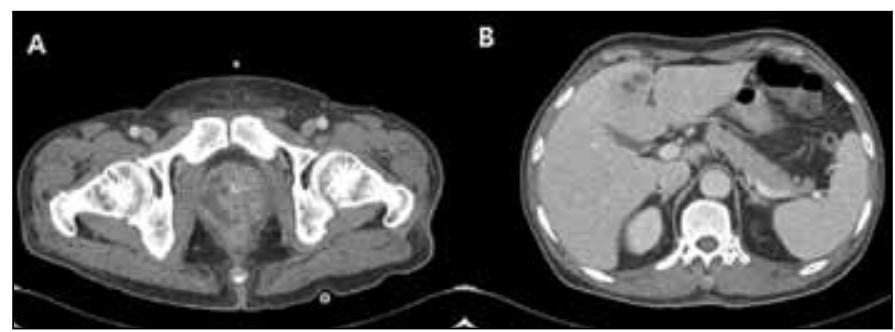

Fig. 2. A: Diffuse enlargement of prostate gland and multifocal fluid collection with peripheral rim enhancement. B: About $2.4-\mathrm{cm}$ sized lobulating low density lesion with peripheral enhancing wall in $\mathrm{S} 4$ of the liver.

disease, such as a prostate abscess, complains of opthalmologic symptoms.

This metastatic phenomenon can be related to the virulence of KP. ${ }^{22}$ In addition to host immunosuppression, Klebsiella specific virulence factors have been implicated in the pathogenicity of this organism. A single intraperitoneal injection of human neutrophils containing phagocytosed KP lead to abscess formation in multiple sites in mice. ${ }^{23}$

This study reported the clinical features of KP-induced prostate abscess based on a small number of patients, which is the main limitation of our study. So far, most of the available data on prostatic abscess are from case reports.

\section{Conclusion}

Among the 6 patients with KP-induced prostate abscess, 4 $(66.7 \%)$ had another site of abscess (pyogenic liver abscess in 3 , and endophthalmitis in 1 patient). We believe that if the causative organism of a prostate abscess was $\mathrm{KP}$, more workup would be needed to rule out the presence of abscess in another organ, especially in the liver. An abdominopelvic CT scan would be a proper imaging modality for this purpose.

Acknowledgement: This work was partially supported by Soonchunhyang University Research Fund.

Competing interests: Dr. J.H. Kim, Dr. Yang, and Dr. T.H. Kim declare no competing financial or personal interests.

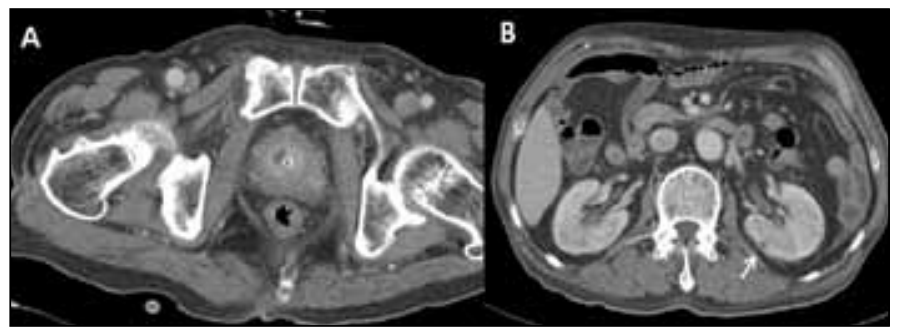

Fig. 3. A: About $1.0 \times 0.7-\mathrm{cm}$ sized low density lesion with fat infiltration in prostate. B: Multiple tiny low density lesion in both kidney suggesting microabscess (arrow). 
Kim et al.

This paper has been peer-reviewed.

\section{References}

1. Arrabal-Polo MA, Jimenez-Pacheco A, Arrabal-Martin M. Percutaneous drainage of prostatic abscess: Case report and literature review. Urol Int 2012;88:118-20. http://dx.doi.org/10.1159/000330934

2. Park SC, Lee JW, Rim JS. Prostatic abscess caused by community-acquired methicillin-resistant Staphylococcus aureus. Int J Urol 2011;18:536-8. http://dx.doi.org/10.1111/i.1442-2042.2011.02774.x

3. Granados EA, Riley G, Salvador J, et al. Prostatic abscess: Diagnosis and treatment. J Urol 1992; 148:80-2.

4. Lim JW, Ko YT, Lee DH, et al. Treatment of prostatic abscess: Value of transrectal ultrasonographically guided needle aspiration. J Ultrasound Med 2000;19:609-17.

5. Weinberger $M$, Cytron $S$, Servadio $C$, et al. Prostatic abscess in the antibiotic era. Rev Infect Dis 1988; 10:239-49. http://dx.doi.org/10.1093/clinids/10.2.239

6. Oliveira P, Andrade JA, Porto HC, et al. Diagnosis and treatment of prostatic abscess. Int Braz I Urol 2003;29:30-4. http://dx.doi.org/10.1590/S1677-55382003000100006

7. Liu KH, Lee HC, Chuang YC, et al. Prostatic abscess in southern Taiwan: Another invasive infection caused predominantly by Klebsiella pneumoniae. J Microbiol Immunol Infect 2003;36:31-6.

8. Jang K, Lee DH, Lee SH, et al. Treatment of prostatic abscess: Case collection and comparison of treatment methods. Korean J Urol 2012;53:860-4. http://dx.doi.org/10.4111/kju.2012.53.12.860

9. Lederman ER, Crum NF. Pyogenic liver abscess with a focus on Klebsiella pneunomiae as a primary pathogen: An emerging disease with unique clinical characteristics. Am J Gastroenterol 2005;100:322-31. http://dx.doi.org/10.1111/i.1572-0241.2005.40310.x

10. Kim JK, Chung DR, Wie SH, et al. Risk factor analysis of invasive liver abscess caused by the K1 serotype Klebsiella pneumoniae. Eur J Clin Microbiol Infect Dis 2009;28:109-11. http://dx.doi.org/10.1007/ s10096-008-0595-2

11. Lin YT, Wang FD, Wu PF, et al. Klebsiella pneumoniae liver abscess in diabetic patients: Association of glycemic control with the clinical characteristics. BMC Infect Dis 2013;13:56. http://dx.doi. org/10.1186/1471-2334-13-56

12. Meddings L, Myers RP, Hubbard J. A population-based study of pyogenic liver abscesses in the United States: Incidence, mortality, and temporal trends. Am J Gastroenterol 2010;105:117-24. http://dx.doi. org/10.1038/aig.2009.614
13. Keller JJ, Tsai MC, Lin CC, et al. Risk of infections subsequent to pyogenic liver abscess: A nationwide population-based study. Clin Microbiol Infec 2013;19:717-22. http://dx.doi.org/10.1111/14690691.12027

14. Tai H-C. Emphysematous prostatic abscess: A case report and review of literature. Infect 2007;54:e51-4. http://dx.doi.org/10.1016/i.jinf.2006.03.033

15. Somuncu I, Sa lam M, Ya ciS, et al. Multiloculated prostate abscess treatment with transrectal ultrasound guided transrectal needle aspiration and lavage with the saline and antibiotic. J Clin Imaging 2003;27:2515. http://dx.doi.org/10.1016/S0899-7071 (02)00546-6

16. Collado A, Palou J, García-Penit J, et al. Ultrasound-guided needle aspiration in prostatic abscess. Urology 1999;53:548-52. http://dx.doi.org/10.1016/50090-4295(98)00570-6

17. Kuo PH, Huang KH, Lee CW, et al. Emphysematous prostatitis caused by Klebsiella pneumonia. J Formos Med Assoc 2007;106:74-7. http://dx.doi.org/10.1016/S0929-6646(09)60219-9

18. Chou YH, Tiu CM, Liu JY, et al. Prostatic abscess: Transrectal color Doppler ultrasonic diagnosis and minimally invasive therapeutic management. Ultrasound Med Bio 2004;30:719-24. http://dx.doi. org/10.1016/i.utrasmedbio.2004.03.014

19. Gautam M, Gandhi A, Rose F. Methicillin-resistant Staphylococcus aureus: Fatal prostatic abscess in an AIDS patient. South Med J 2008;101:449. http://dx.doi.org/10.1097/SMJ.0b013e318167badd

20. Goyal NK, Goel A, Sankhwar $S$, et al. Transurethral resection of prostate abscess: Is it different from conventional transurethral resection for benign prostatic hyperperplasia? ISRN Urology 2013;109505.

21. Pope JV, Teich DL, Clardy P, et al. Klebsiella pneumonia liver abscess: An emerging problem in North America. J Emerg Med 2011;41:e103-5. http://dx.doi.org/10.1016/i.jemermed.2008.04.041

22. Lim KH, Tan YM, Chow PHK. Liver abscess metastasizing to prostate and lung. J R Soc Med 2002;95:5545. http://dx.doi.org/10.1258/irsm.95.11.554

23. Kashani AH, Eliott D. The emergence of Klebsiella pneumonia endogenous endophthalmitis in the USA: Basic and clinical advances. J Ophthalmic Inflamm Infect2013;3:1-6. http://dx.doi.org/10.1186/18695760-3-28

Correspondence: Dr. Won Jae Yang, Department of Urology, Soonchunhyang University Seoul Hospital, 59, Daesagwan-ro, Yongsan-gu, Seoul, 140-743, South Korea; wonija@schmc.ac.kr 